\title{
Endovascular Treatment of intracranial Pial Arteriovenous Fistula in
}

\section{Pediatric Patient}

\author{
Marco Zenteno ${ }^{1}$, Jorge Balderrama ${ }^{1}$, Alfredo Montenegro ${ }^{1}$, Carmen Vargas ${ }^{1}$, Beatriz Mendez ${ }^{1}$, Miguel Angel Nieto ${ }^{2}$, Cindy Díaz ${ }^{*}$ \\ ${ }^{1}$ Department of Endovascular Therapy, Universidad Nacional Autonoma de Mexico, Instituto Nacional de Neurologia y Neurocirugia Manuel \\ Velasco (INNN) \\ ${ }^{2}$ Department of Pediatric Anesthesiology, Universidad Naval, Escuela de Posgrados en Sanidad Naval, Universidad Nacional Autónoma de Mexico, \\ Instituto Nacional de Pediatría
}

* Corresponding Author: Cindy Díaz, Department of Endovascular Therapy, Universidad Nacional Autonoma de Mexico, Instituto Nacional de Neurologia y Neurocirugia Manuel Velasco (INNN)

Received date: February 03, 2021; Accepted date: March 12, 2021; Published date: March 19,2021

Citation: Marco Zenteno, Jorge Balderrama, Alfredo Montenegro, Carmen Vargas, Beatriz Mendez, Miguel Angel Nieto, Cindy Díaz (2021)

Endovascular Treatment of intracranial Pial Arteriovenous Fistula in Pediatric Patient J. Neuroscience and Neurological Surgery. 8(2);

DOI:10.31579/2578-8868/167

Copyright: @ 2021 Cindy Díaz, This is an open-access article distributed under the terms of The Creative Commons Attribution License, which permits unrestricted use, distribution, and reproduction in any medium, provided the original author and source are credited

\begin{abstract}
:
Pial arteriovenous fistula (AVFs) are a rare, although clinically significant, vascular anomaly affecting the pediatric population, consisting of a high-flow connection between one or more pial arteries and the venous. In children, the clinical presentation varies according to age, can present with high output cardiac failure, macrocrania, neurodevelopmental delay, seizures, venous infarctions due to venous stenosis or hemorrhage secondary to venous thrombosis. The treatment, is a multimodal approach, most commonly endovascular, is effective in the obliteration of AVF. A case is presented of a previously healthy infant boy with neurological deficit arising from pAVF of the middle and posterior cerebral artery. The pAVF was successfully treated with endovascular occlusion.

Keywords: endovascular treatment; pial arteriovenous fistula; paediatric patient
\end{abstract}

\section{Introduction}

Pediatric pial arteriovenous fistula (pAVF) are rare vascular lesions of the CNS, characterized by direct arterial connections to a pial venous channel, without an intervening nidus. [1] Once thought to be a type of arteriovenous malformation (AVM), they are now recognized as a distinct vascular anomaly. It represent approximately $4 \%$ of pediatric cerebral vascular anomalies comprised primarily of arteriovenous malformations (AVMs). The etiology of pAVF is incompletely understood. Theories include the development of dAVF as a sequela of venous sinus thrombosis or secondary to venous sinus hypertension. Presenting symptoms include mass effect, especially if a venous pouch is associated, hemorrhage, seizures, cardiac failure and/or venous hypertension. [2] The high rate of arteriovenous shunting and deep venous drainage decreases the efficacy of microsurgical treatment, and nowadays, endovascular treatment of PAVF is preferable.3Transarterial embolization is the primary method of treatment, although open surgery and radiosurgery have also been employed or a combination. [4]

\section{Case Report}

A healthy infant male, suddenly develops headache in thunderclap with altered state of consciousness, presented to an emergency room in coma with anisocoria. Brain $\mathrm{CT}$, revealed acute intraparenchymatous left subdural hematoma, giant vascular dilatation in left temporal lobe (figure 1).
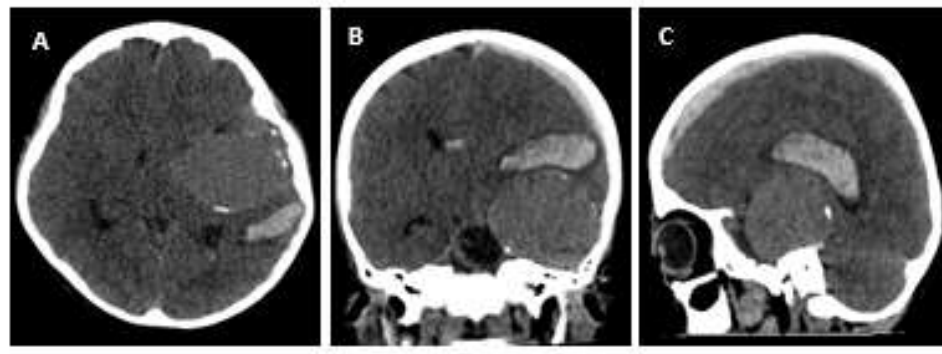

Figure 1: Simple cranial CT: A. Axial, B.Coronal, C, Sagittal: frontal-temporal left intraparenchymatous hematoma, left hemispheric subdural hematoma with mass effect, hyperdense rounded image with well-defined edges with a calcified wall. 
Surgery was performed, with left decompressive craniotomy, acute and intraparenchymatous subdural hematoma was drained, vessels with active bleeding from vascular malformation were identified, $5 \mathrm{~mm}$ clip was placed, severe brain edema was also observed. An MRI of the brain revealed a giant vascular venous dilatation at left temporal level, with arterio-venous short circuit (figure 2).
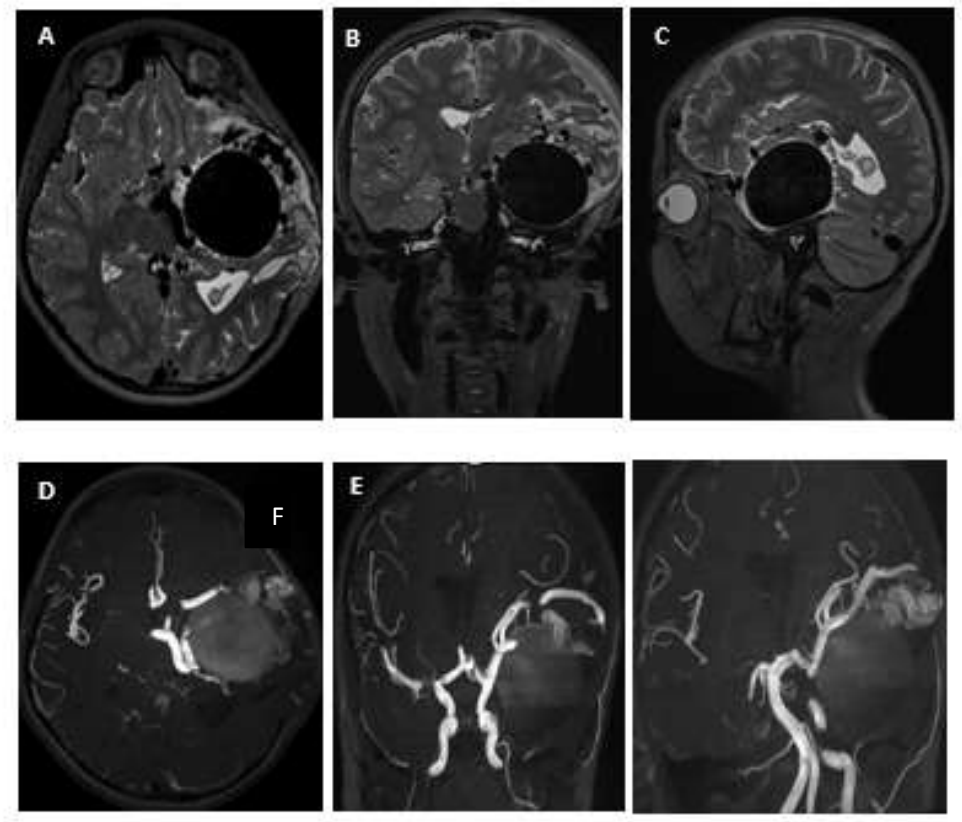

Figure 2: Angiographic protocol MRI (Magnetic resonance imaging): 2 weighed images in axial (a), coronal (b) and sagital(c) planes. Multiple serpentine vascular structures with signal void. A big venous varix with diameter of $51.3 \mathrm{mmX} 53.1 \mathrm{~mm}$. 3DTOF axial (D) coronal (E) shows feeders from MCA's (middle cerebral artery) and temporal branches lower trunk and the left PCA (posterior cerebral artery) $(F)$.

A giant AVF was suspected, and definitive treatment by an endovascular approach was planned. The treatment was performed 90 days after the initial presentation. Diagnostic angiogram demonstrated a multi-hole pial AVF with venous dilation of $54 \times 56 \mathrm{~mm}$ which was supplied by enlarged branches of the left posterior cerebral artery (PCA), middle cerebral artery's medial and posterior basal temporal branches' lower trunk. Venous outflow drained to a dilated Labbé vein, left lateral sinus and lateral tentorial sinus (figure 3).
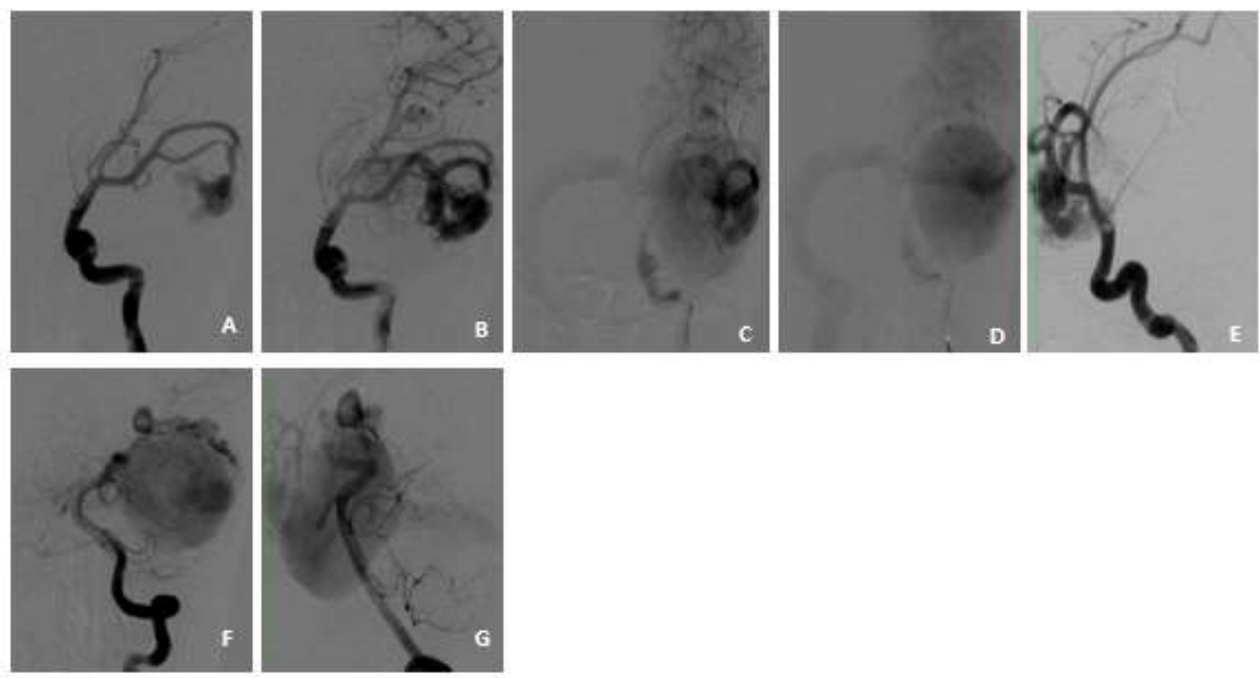

Figure 3: Left internal carotid artery: AP (A-D) and lateral (E) DSA (Digital subtraction angiography), demonstrating multi-hole pial AVF with venous dilation $54 \times 56 \mathrm{~mm}$, supplied by MCA's lower trunk mean and posterior basal temporal branches. Venous flow to a dilated Labbé Vein, right lateral sinus and lateral tentorial sinus, venous outflow was into a dilated Labbé vein and right lateral sinus and lateral tentorial sinus. Left vertebral artery: $A P(F)$ and lateral $(G)$ DSA, afferences: Posterior cerebral artery branches $(P C A)$. 
Definitive treatment by an endovascular approach was planned.

Embolization were performed with $5 \mathrm{~F}$ introducer in the right femoral artery, balanced general anesthesia was used, with non-invasive basic monitoring, anesthetic induction with fentanyl ( $3 \mathrm{mcg} / \mathrm{kg}$ ), lidocaine (1 $\mathrm{mg} / \mathrm{kg}$ ) and propofol $(3 \mathrm{mg} / \mathrm{kg})$, the anesthetic was maintenance with Sevoflurane $2 \mathrm{~V} \%$ to maintain $0.8 \mathrm{MAC}$, the patiente had a stable hemodynamic behavior, due to the high flow characteristics of the fistulae and to avoid the passage of the embolizing material, blood pressure had to be managed dynamically, with intermittent and brief periods of hypotension, administering propofol in bolus $1 \mathrm{mg} / \mathrm{kg}$ in 3 times, reaching mean arterial pressures of $62-64 \mathrm{mmHg}$, later to corroborate the embolization of the fistulae, blood pressure was increased for a period no longer than 15 minutes, for which epinephrine was administered by intravenous infusion at $0.02-0.04 \mathrm{mcg} / \mathrm{kg} / \mathrm{min}$ until gradually reaching mean arterial pressures of $68-69 \mathrm{mmHg}$, suspending the infusion at the end of said period of time.
Embolization was undertaken via a $4 \mathrm{~F}$ Terumo guide catheter (Terumo Medical Corp, Somerset, New Jersey, USA) and Excelsior SL-10 microcatheter (Stryker Neurovascular). Traxcess 14 guidewire (Microvention) and Mirage ${ }^{\mathrm{TM}} .008$ " hydrophilic guidewire (Medtronic). With the guiding catheter in the left internal carotid artery, a microcatether was placed on MCA's the lower trunk and the first embolization was performed. $1 \mathrm{cc}$ of N-butyl cyanoacrylate (NBCA), was safely injected to achieve partial fistulae occlusion (figure 4). Then the guiding catheter is placed in the left vertebral artery, a microcatether was placed in the PCA ipsilaterally and embolization was performed with $1 \mathrm{cc}$ (NBCA) was safely injected to achieve parcial occlusion of the fistulae with passage of embolizing material inside the venous varix, so the microcateter is repositioned (figure 4) in the vein foot and a embolization is performed with $0,8 \mathrm{cc}$ (NBCA) to achieve complete occlusion (figure 4). On the final angiographic control, observed normal anterior circulation was preserved and venous drainage had returned to normal.
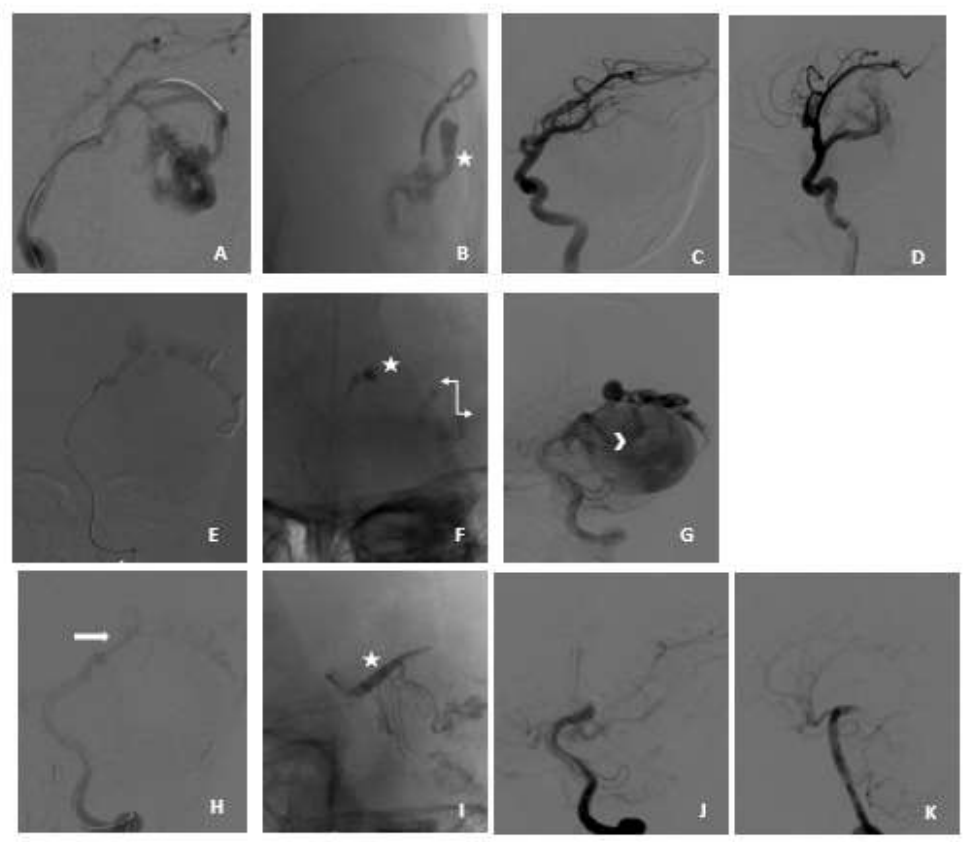

Figure 4: Left cerebral middle artery: $A P(A-D)$, demonstrating microcatether was placed in the lower trunk of the left middle cerebral artery (A) and first embolization was performed con $N$-butyl cyanoacrylate (NBCA) (star) was safely injected (B) to achieve parcial occlusion of the fistula $(C$-D). Left posterior cerebral artery (PCA), AP (E-G) DSA, demonstrating a microcatether placed in the posterior cerebral artery ipsilateral (E), embolization was performed con $N$-butyl cyanoacrylate (NBCA) (star) was injected $(F)$, with reflux of embolizing material inside the venous varix $(F-G)$ double arrow $(F)$ and mitre $(G)$, so the microcateter is repositioned.

Left posterior cerebral artery (PCA), AP (H-K) DSA, demonstrating a microcatether repositioned in the vein $(H)($ arrow), second embolization was performed con N-butyl cyanoacrylate (NBCA) (star) was injected (I), achieve occlusion of the fistula, DSA post-embolization control, AP (J), lateral $(K)$.

The patient was transferred to the pediatric intensive care unit where he recovered for a week without neurological deficit, then hospital was discharge. After a 3 month clinical and MRI control, a complete thrombosis and a reduction of the venous puch was seen. At the time of the last follow-up, the patient was completely asymptomatic without any neurological deficit.

\section{Discussion}

Pial arteriovenous fistula (pAVF) are rare lesional vascular anomalies that pose a high risk of intracranial hemorrhage across all age groups, including children.5 pAVF does not demonstrate a nidus, is supplied from the pial or cortical arteries, and is characterized by abnormal direct highflow connection between an arterial feeding vessel and draining vein, which has a significant impact on the cerebral blood flow. [6] The reported incidence in literature varies: $3 \%$ (13 of $500 \mathrm{AVM})$ in one to $4.8 \%$ (12 of 251 AVM) in another series. [7] Our patient presented with signs and symptoms of a mass effect from a dilated venous pouch and intracerebral hemorrhage. 
Embryologically, a fistulae is a likely explanation of abnormally dilated capillary nets that shunt blood from developing arteries to veins. In congenital pial fistulae, the fistulous connection of the dilated capillary nets that develop at this early stage of vascular development randomly persisted with the failure of the structured and more mature capillary network to develop. 8 Most of the arteries feeding the pial AVF open into a single ectatic draining vein frequently associated with large to giant venous varix, and their presence is determined by the high flow across the shunt against venous outflow obstruction. [9] Lasjaunias et al classified between three types of dural arteriovenous shunts that appear in children: (i) dural sinus malformations (congenital malformations of sinus development with giant pouches or malformations of the jugular bulb where thrombosis or occlusion of the sinus outflow is a central clinical concern), (ii) infantile-type dural arteriovenous shunts (high flow, often multifocal lesions with patent sinuses and induced pial arteriovenous shunts) and (iii) adult-type dural arteriovenous shunt. [10]

The treatment of PAVF include multidisciplinary approach, is important to evaluate the location and degree of lesion complexity. [12] The goal of treatment in cortical AVF in young children, even when asymptomatic, is rapid control of the shunt, because of high mortality and worse neurocognitive prognosis when managed conservatively. [10] Increased endovascular experience leading to evolution of techniques and the less invasive nature of the procedure have made endovascular treatment the preferred treatment modality (obliteration rate of $86.5 \%$ ), offered only to selected superficial fistulae. [13] Endovascular treatment can be done in a few stages to reduce the risk for postoperative hemorrhage by preventing normal perfusion pressure breakthrough in high-flow lesions. [14]

Embolic agents such as NBCA, coils, Onyx 34 have been used for the successful occlusion of the fistulae. Techniques have evolved to counter these such as the use of tandem balloon, balloon-assisted NBCA embolization. [15]

\section{Conclusion}

Pial AVF is a rare disease caused by a missed step in vascular development during the early embryonic stage. Treatment, most commonly endovascular, is effective in the obliteration of pAVF in children, with most patients experiencing substantial and durable improvement of clinical symptoms. In our experience, transarterial endovascular embolization using NBCA with or without using coils on the venous side to attain flow control is the treatment of choice with low morbidity. Follow-up is mandatory to look for recanalization and reactive angiogenesis with reopening of the shunt and de-novo dural AVF.

\section{Conflict of interest:}

\section{References}

1. Hoh BL, Putman CM, Budzik RF, et al. Surgical and endovascular flow disconnection of intracranial pial singlechannel arteriovenous fistulae. Neurosurgery 2001;49:1351e63.

2. Weon Y, Yoshida Y, Sachet M, et al. Supratentorial cerebral arteriovenous fistulas (AVFs) in children: review of 41 cases with 63 non choroidal single-hole AVFs. Acta Neurochir (Wien) 2005;147:17e31.

3. Andreou A, Ioannidis I, Nasis N. Transarterial balloon-assisted glue embolization of high-flow arteriovenous fistulas. Neuroradiology 2008;50(03):267-272

4. Lasajaunias $P$, ter Brugge $K$, Berenstein A. Surgical neuroangiography: clinical and interventional aspects in children. Leipzig: Springer, 2006

5. Lv X, Jiang C, Li Y, Yang X, Wu Z. Clinical outcomes of endovascular treatment for intracranial pial arteriovenous fistulas. World Neurosurg 2010;73(04):385-390

6. Weon YC, Yoshida Y, Sachet M, et al. Supratentorial cerebral arteriovenous fistulas (AVFs) in children: review of 41 cases with 63 non choroidal single-hole AVFs. Acta Neurochir (Wien) 2005;147:17e31.

7. Srinivasan P, Naoki T, et al. Development, clinical presentation and endovascular management of congenital intracranial pial arteriovenous fistulas. J NeuroIntervent Surg 2013;5:184-190. doi:10.1136/neurintsurg-2011-010241

8. Wedderburn CJ, van Beijnum J, Bhattacharya JJ, et al. Outcome after interventional or conservative management of unruptured brain arteriovenous malformations: a prospective, populationbased cohort study. Lancet Neurol 2008;7:223e30.

9. Nelson PK, Nimmy Y, Lasjaunias P, et al. Endovascular embolization of congenital intracranial pial arteriovenous fistulas. Neuroimaging Clin N Am 1992;2:309e17. Tomlinson FH, Rufenacht DA, Sundt TM Jr, et al. A

10. Lasjaunias PL, Berenstein A, Terbrugge KG. Surgical neuroangiography. 2nd edn. Berlin: Springer, 2001.

11. Yang WH, Lu MS, Cheng YK, et al. Pial arteriovenous fistula: a review of literature. Br J Neurosurg 2011;25:580e5.

12. Paramasivam S, Toma N, Niimi Y, Berenstein A. Development, clinical presentation and endovascular management of congenital intracranial pial arteriovenous fistulas. J Neurointerv Surg 2013;5(03):184-190

13. Lv X, Li Y, Jiang C, et al. Endovascular treatment of brain arteriovenous fistulas. AJNR Am J Neuroradiol 2009;30:851e6.

14. Andreou A, Ioannidis I, Nasis N. Transarterial balloon-assisted glue embolization of high-flow arteriovenous fistulas. Neuroradiology 2008;50:267e72.

15. Nishijima M, Takaku A, Endo S, et al. Etiological evaluation of dural arteriovenous malformations of the lateral and sigmoid sinuses based on histopathological examinations. J Neurosurg 1992;76:600e6.

None 
(c) (i)

This work is licensed under Creative

Commons Attribution 4.0 License

To Submit Your Article Click Here: Submit Manuscript

DOI: $10.31579 / 2578-8868 / 167$
Ready to submit your research? Choose Auctores and benefit from:

* fast, convenient online submission

* rigorous peer review by experienced research in your field

* rapid publication on acceptance

* authors retain copyrights

* unique DOI for all articles

* immediate, unrestricted online access

At Auctores, research is always in progress.

Learn more www.auctoresonline.org/journals/neuroscience-andneurological-surgery 